\title{
Age, Period and Cohort Analysis of Rates of Emergency Department Visits Due to Pneumonia in Taiwan, 1998-2012
}

This article was published in the following Dove Press journal: Risk Management and Healthcare Policy

\author{
Shin-Hong Chen,' I-Shiang \\ Tzeng, (1D) ${ }^{2-4}$ Chou-Chin \\ Lan, (iD) ${ }^{5,6}$ Jau-Yuan Chen, ${ }^{7}$ \\ Chau Yee $\mathrm{Ng}$, (D) ${ }^{8}$ Yao-Chin \\ Wang, (1D ${ }^{9}$ Wen-Lin Su, (1D ${ }^{5}$ \\ Giou-Teng Yiang, (ID) ${ }^{10}$ Tsu- \\ Yi Chen, ${ }^{10}$ Chih-Wei Wu, ${ }^{5}$ \\ Po-Chun Hsieh, "' Chan-Yen \\ Kuo, (D) ${ }^{4}$ Meng-Yu Wu (D) ${ }^{10}$
}

'Department of Education and Research, Taiwan Adventist Hospital, Taipei I0556, Taiwan; ${ }^{2}$ Department of Statistics, National Taipei University, Taipei 10478, Taiwan; ${ }^{3}$ Department of Applied Mathematics; Department of Exercise and Health Promotion, Chinese Culture University, Taipei III I4, Taiwan; ${ }^{4}$ Department of Research, Taipei Tzu Chi Hospital, Buddhist Tzu Chi Medical Foundation, New Taipei City 23।42, Taiwan; ${ }^{5}$ Division of Pulmonary Medicine, Taipei Tzu Chi Hospital, Buddhist Tzu Chi Medical Foundation, New Taipei City 23।42, Taiwan; ${ }^{6} \mathrm{~S}$ chool of Medicine, Tzu-Chi University, Hualien 97004, Taiwan; ${ }^{7}$ Department of Family Medicine, ChangGung Memorial Hospital at Linkou, Taoyuan 33305, Taiwan; ${ }^{8}$ Department of

Dermatology, Drug Hypersensitivity Clinical and Research Center, Chang Gung Memorial Hospital, Taipei, Linkou and Keelung 10507, Taiwan; ${ }^{9}$ Department of Emergency Medicine, Min-Sheng General Hospital, Taoyuan 33044, Taiwan; ${ }^{10}$ Department of Emergency Medicine, Taipei Tzu Chi Hospital, Buddhist Tzu Chi Medical Foundation, New Taipei City 23142, Taiwan; "'Department of Chinese Medicine, Taipei Tzu Chi Hospital, Buddhist Tzu Chi Medical Foundation, New Taipei City 23I42, Taiwan

Correspondence: I-Shiang Tzeng No. 289, Jianguo Road, Xindian Dist, New Taipei City 23I, Taiwan

Tel +886-2-6628-9779 ext. 3119

Email istzeng@gmail.com
Background: Emergency room (ER) physicians need to face clinically suspected pneumonia patients in the front line of medical care and must do to give major medical interventions if patients show severity in pneumonia.

Methods: The data of pneumonia-related ER visit rates were categorized based on the International Classification of Disease (ICD) Codes (480-486) between 1998 and 2012. We use an age-period-cohort (APC) model to separate the pneumonia-related ER visit rates to identify the effects of age, time period, and cohort for a total of 1,813,588 patients.

Results: The age effect showed high risk for pediatric and elder populations. There is a significant increasing period effect, which increased from 1998 to 2012 . The cohort effect tended to show an oscillation from 1913 to 1988 and the reverse in a recent cohort. Furthermore, the visit rate of pneumonia showed an increase from 1998 to 2012 for both genders.

Conclusion: Age is a risk factor for pneumonia-related ER visits, especially for children and adolescents and older patients. Period and cohort effects were also found to increase the pneumonia visit rates. An APC model used to provide an advance clue for trend of pneumonia-related ER visit rates diversified.

Keywords: age-period-cohort, age-adjusted emergency rooms visit rates, pneumonia

\section{Background}

Seasonal influenza infects millions of people worldwide annually. For example, in the US, it infects about $5-20 \%$ of the population every year, resulting in over 200,000 hospitalizations. ${ }^{1}$ There are several complications of influenza that contribute to adult mortality. One of the serious complications of influenza is pneumonia. $^{2}$ Moreover, pneumonia had increased the disease severity in children hospitalized with influenza infection. For the last recorded observation, the median pneumonia mortality rate was $19.8 / 100,000$ and $6.9 / 100,000$ across the European Union (EU) for men and women, respectively. ${ }^{3}$ The mortality rate of pneumonia is much higher in the older age groups than in the younger age groups, and those aged under 5 years also had a slightly higher mortality rate than the younger age groups.

In recent decades, both pneumonia prevalence and incidence have been increasing worldwide, primarily due to the increase of aged population. ${ }^{4}$ However, the worldwide economic globalization could also have contributed to a significant increase in pneumonia burden. ${ }^{5}$ Pneumonia remains the leading cause of death in developing countries and is the most common reason for hospital admission. ${ }^{6}$ 
Emergency room (ER) physicians need to dispose clinically suspected pneumonia patients in the front line of medical care and must give major medical interventions if patients show symptoms of severe pneumonia. Several predictive factors can aid ER physicians in the diagnosis of pneumonia, including the presence of hypoxemia and chest retraction score. ${ }^{7}$ Pneumonia is an infection caused by invasion of pathogens in the lower respiratory tract, inducing a pulmonary inflammatory response. Bacteria was still an important cause of pneumonia in many developing countries where no vaccine was introduced. ${ }^{8}$ Among the virus, the respiratory syncytial virus (RSV), influenza virus, human parainfluenza virus (HPIV), human rhinovirus (HRV), and human adenovirus (HAdV) are usually detected in children suffering from pneumonia. ${ }^{8}$ However, in case of early pneumonia, the presence of certain pathogens including molds, bacteria, viruses, and tuberculosis would make it difficult to diagnose the inflammatory response. Many therapeutic innovations in treatment have been demonstrated in experimental preclinical studies which improved the outcome of patients with severe pneumonia due to loss of the innate immune system leading to lung injury and its systemic sequelae attributed to destroy vascular barrier function, harmful hyperinflammation, and microcirculatory failure. ${ }^{9}$ In 2016 , pneumonia ranks the third leading cause of death in men and the fifth leading cause of death in women in Taiwan. The number of deaths due to pneumonia increased from 3619 in 1997 to 12,212 in 2016, with an annual growth of about $6.26 \% .{ }^{10}$ Based on direct observations of the longitudinal trends in the number of pneumonia-associated deaths from 1997 to 2016 in Taiwan, it is important for physicians to observe a sustained trend in the hospitals' capacity to manage patients. In addition, the emergency physicians' capacity to provide medical services has not only been linked with patients' health status, but it has also been associated with the labor provided for critical inflammatory diseases.

Based on our knowledge in epidemiology, the age-period -cohort (APC) model can be used to represent the secular trend in disease incidence or mortality. ${ }^{11} \mathrm{~A}$ three-factor multiplicative model separates the age, period, and cohort effects from the trends in disease incidence or rates of a particular event. Age effects are associated with the outcome of time, resulting in the changes in the number of outcomes (deaths caused by cancer). Period effects can affect all ages simultaneously over time. Birth cohort effects involve changes across groups with the same birth year who presented the same outcome during the same period. These effects separated by the APC model may provide epidemiologists with important insights on identifying health determinants, hypothesis testing, or potential sources of heterogeneities in the literature. However, less effort has been made previously to investigate these effects of rates of ER visits due to pneumonia by the APC model.

This study estimated the longitudinal trends in the rate of pneumonia-related ER visits by utilizing an aggregated report obtained from a claims database of the National Health Insurance program in Taiwan. The pneumoniarelated ER visit rates were separated to identify the age, period, and cohort effects, and examine whether these effects varied by sex.

\section{Methods \\ Data Sources}

We proposed a retrospective study. Data on pneumoniarelated ER visit rates from 1998 to 2012 among men and women in Taiwan were collected. These data were obtained from patients' individual health records, which were provided by the Health Promotion Administration, Ministry of Health and Welfare (MOHW), Taiwan. The study period determined due to the National Health Insurance has implemented the global budget system since 1998 and available data end in 2012. Pneumonia-related ER visit rates were categorized based on the International Classification of Disease (ICD) Codes (480-486). The visit data included 185 -year age groups $(0-4,5-9,10-14,15-19,20-24$, 25-29, 30-34, 35-39, 40-44, 45-49, 50-54, 55-59, 60-64, 65-69, 70-74, 75-79, 80-84, and 85+), three 5-year time periods (1998-2002, 2003-2007, and 2008-2012), and 20 birth cohorts (1913, 1918, 1923, 1928, 1933, 1938, 1943, 1948, 1953, 1958, 1963, 1968, 1973, 1978, 1983, 1988, 1993, 1998, 2003, and 2008). Using the aforementioned data, we calculated the age-specific and age-adjusted visit rates using the 2000 World Standard Population. ${ }^{12}$ The present study was approved by the Institutional Review Board of Chang Gung Memorial Hospital (No. 103-6269B). Informed consent was waived by the review board in this study due to secondary data analysis.

\section{Statistical Analysis}

The log-linear Poisson model showed that each factor has an additive effect on the log rate:

$$
k=j-i+I,
$$

where $\mu$ represents the intercept, $\alpha_{i}$ the age effects, $\beta_{j}$ the period effects, and $\gamma_{k}$ the cohort effects. The age, period, 
and cohort effects were satisfied as following constraint, $\sum \alpha_{i}=\sum \beta_{j}=\sum \gamma_{k}=0$.

Estimates of ${ }^{j}$ the parâmeters of the above APC model were used to explore the pneumonia-related ER visit rates. The maximum likelihood estimation was utilized for estimates of the parameters, which were used by free $\mathrm{R}$ software (version 3.4 .3$)^{13}$ for analysis.

\section{Overdispersion Test}

The overdispersion analysis via free $\mathrm{R}$ software was performed to investigate the overdispersion for Poisson model with the overdispersion test. ${ }^{14}$ The procedure can be conveniently tested using the R package "AER".

\section{Patient and Public Involvement}

In this study, we confirmed that patients were not involved in development of the study design, research questions, and interpretation of results corresponding to be written in the manuscript.

\section{Results}

The overdispersion analysis showed there is significance of overdispersion $\left(P=1.474 \times 10^{-04}\right.$ and $1.096 \times 10^{-06}$ for males and females, respectively), which means contain strongly against the assumption of equidispersion. In total, there were 1,813,588 pneumonia-related ER visits made by newborn to older patients, more than $58 \%$ of which were men (58.3\%, Table 1). Those aged $0-19$ years and middle-aged patients (aged 40-64 years) contributed $45.1 \%(\mathrm{~N}=818,208)$ and $14.6 \%(\mathrm{~N}=264,380)$ of these pneumonia-related ER visits, respectively, whereas the elderly (age $\geq 65$ years) was the secondly predominant source population $(\mathrm{N}=561,176,30.9 \%$, Table 1). A predominance of male patients aged 0-19 years was consistently observed across the three calendar periods (Table 1).

Figure 1 shows the age effects. The age effects are quite notable for both sexes. In males, the age effect increased from 3.65 (for the youngest age group: 0-4 years) to 9.46 (for the oldest age group: $85+$ years), with the visit rate increasing by $\exp (9.46) / \exp (3.65)=333.62$-fold. In females, the age effect increased from 3.98 to 7.89 (visit rate increasing by 49.90 -fold). To the age effects on pneumonia-related ER visit rates, it means the 0-4 years group is 333.62 (49.90) times the visit rates of the $85+$ years group for males (females).

Period effects are presented in Figure 2. In males, the period effect increased from -0.32 (for the period of 1998-2002) to 0.39 (for the period of 2008-2012), with the visit rate increasing by 2.03 -fold. In females, the period effect increased from -0.27 to 0.35 (visit rates increasing by 1.85 -fold). To the period effects on pneumonia-related ER visit rates, it means the period of 2008-2012 is 2.03 (1.85) times the visit rates of the period of 1998-2002 for males (females).

Figure 3 presents the cohort effects. In males, the cohort effect decreased from 1.68 (for the highest-risk birth cohort: mid-cohort year of 1933) to 0.80 (for the most recent birth cohort: mid-cohort year of 2008), with the visit rates decreasing by about two-thirds, which is $41 \%$ of the peak value. However, in females, the increase in the recent cohort effects was striking. The cohort effect increased from 1.37 to 1.75 (with visit rates increasing to $46 \%$ of the peak value).

Figure 4 presents the logarithms of the age-specific 5-year pneumonia-related ER visit rates (age groups of $0-4,5-9, \ldots, 85+$ ) for males and females corresponding to patients' birth cohorts, respectively. We also present

Table I Case Numbers of ER Patient Visits from Pneumonia

\begin{tabular}{|c|c|c|c|c|c|}
\hline \multirow[t]{3}{*}{ Characteristics } & Overall & $1998-2002$ & 2003-2007 & $2008-2012$ & \multirow[t]{3}{*}{$P$-value } \\
\hline & Total (\%) & Total (\%) & Total (\%) & Total (\%) & \\
\hline & $N=|, 8| 3,588$ & $N=462,983$ & $N=547,375$ & $N=803,230$ & \\
\hline \multicolumn{6}{|l|}{ Gender } \\
\hline Male & I,057,4| (58.3) & $269,980(58.3)$ & $321,978(5 I . I)$ & $465,460(57.9)$ & $<0.000$ I* \\
\hline Female & $756, \mid 70(4 \mid .7)$ & I93,003 (4I.7) & 225,397 (48.9) & $337,770(42.1)$ & \\
\hline \multicolumn{6}{|l|}{ Age } \\
\hline $0-19$ & $818,208(45.1)$ & $235,627(50.9)$ & $261,249(47.7)$ & $321,332(40.0)$ & $<0.000$ I* \\
\hline $20-39$ & $169,824(9.4)$ & $45,164(9.7)$ & $53,065(9.7)$ & 71,595 (8.9) & \\
\hline $40-64$ & $264,380(14.6)$ & $62,356(13.5)$ & $73,438(13.4)$ & $128,586(16.0)$ & \\
\hline$\geq 65$ & $561,176(30.9)$ & $119,836(25.9)$ & I59,623 (29.2) & $28 I, 717(35.1)$ & \\
\hline
\end{tabular}

Note: *Significant at the 0.05 level for Chi-square test to test for independence between characteristics and time periods. 
Males

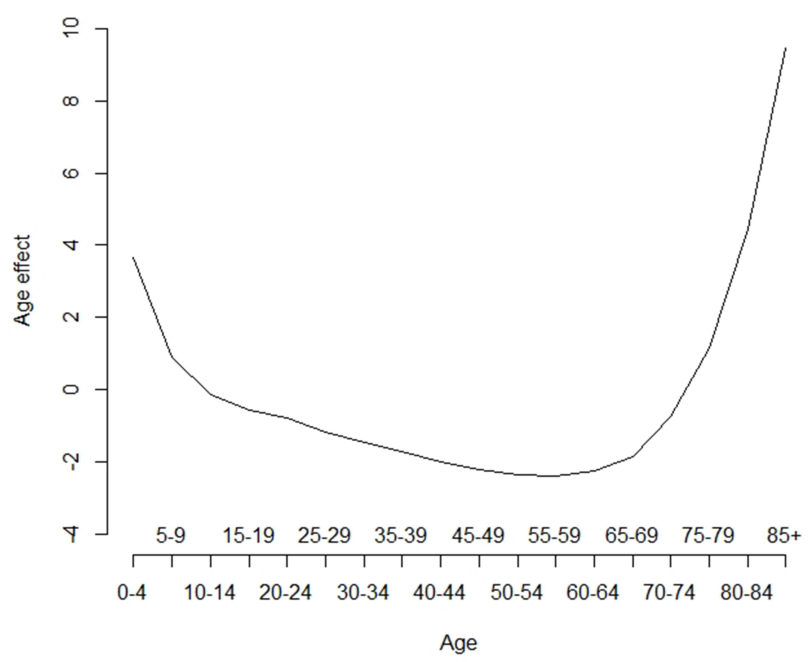

Females

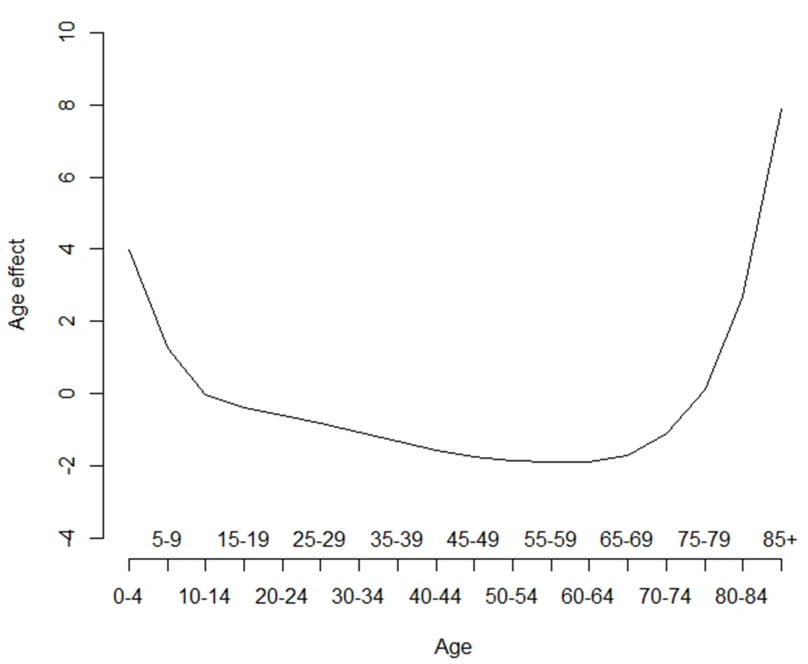

Figure I The age effects of pneumonia-associated ER visits rates for males and females in Taiwan, 1998-20I2.

Males

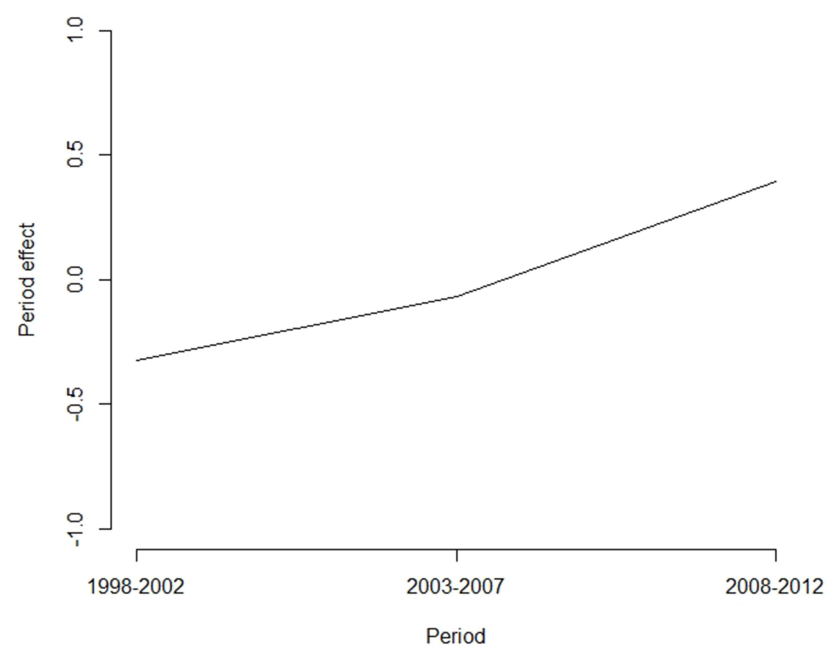

Females

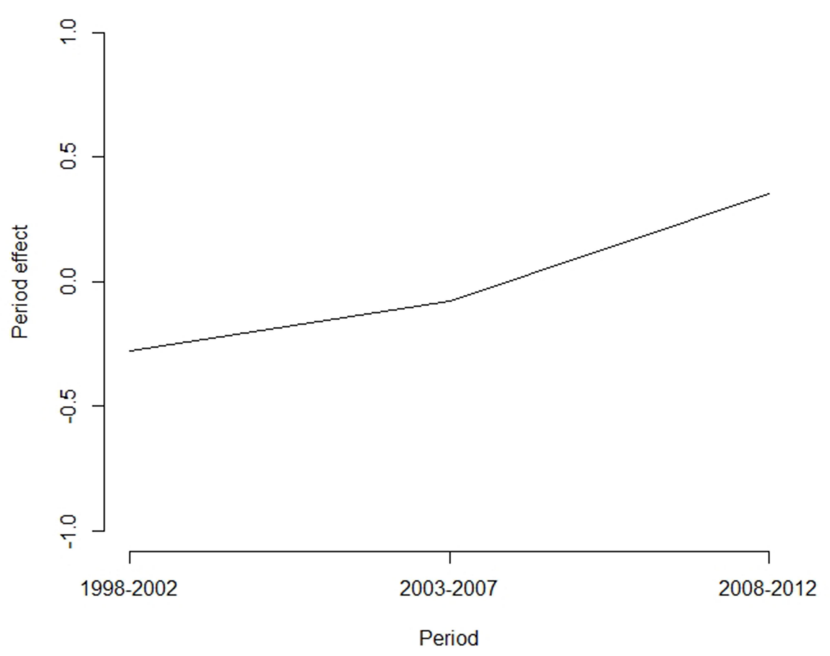

Figure 2 The period effects of pneumonia-associated ER visits rates for males and females in Taiwan, 1998-2012.

time period patterns for each age group with three points (ie, three 5-year time periods: 1998-2002, 2003-2007, and 2008-2012) connected as a solid line.

\section{Discussion}

A birth cohort-driven increase in visit rates due to pneumonia was observed (Figure 4), which was stronger in women than in men in the recent birth cohort. More specifically, there was reprography "N shape" of birth cohort curves for the pneumonia while statistical apparent exploration for pneumonia-related visit rates.
Previous studies have examined the overall trends in pneumonia-related ER visits. The visit rate for pneumonia may have an increased trend during the longitudinal period, ${ }^{15,16}$ which has seen improvements over decades in diagnosis and awareness. ${ }^{17,18}$ Similarly, we observed an increasing trend in the rates of pneumonia-related ER visits within the study period (Figure 2), which continued to increase. With regard to age effects, children and adolescents and older adults had higher pneumonia-related ER visit rates (Figure 2). To shed more insight on these effects would influence current clinical practice. We provided the estimation of future visit cases and the comparison with ER 
Males

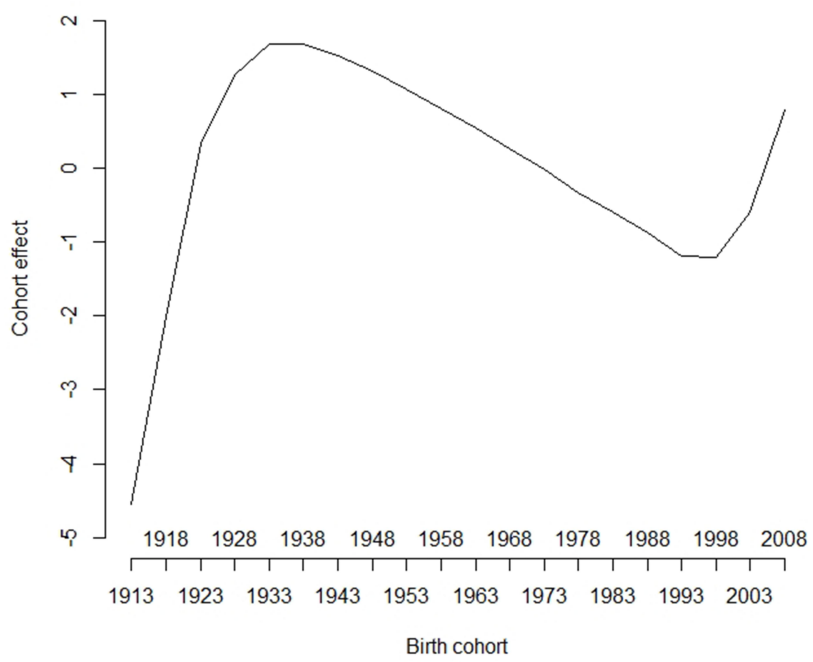

Females

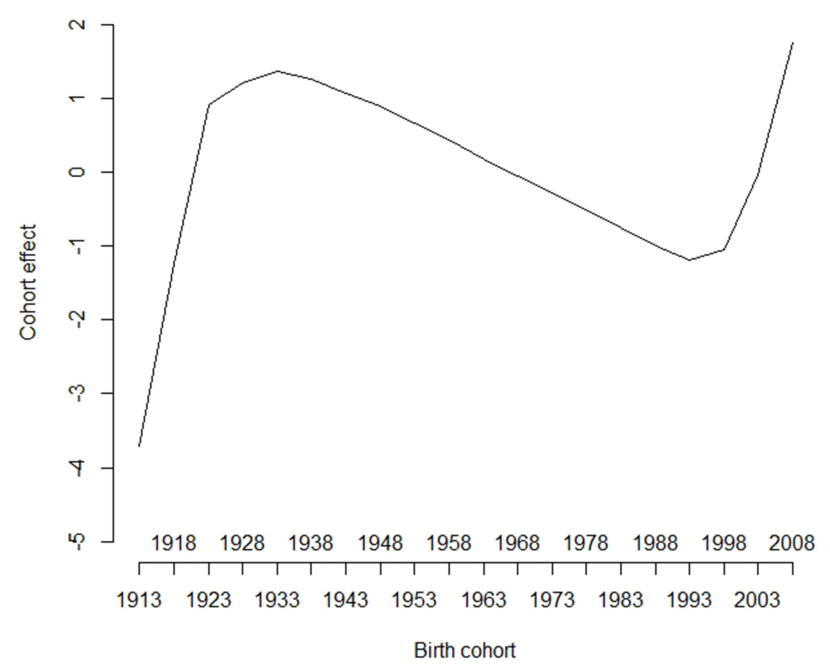

Figure 3 The cohort effects of pneumonia-associated ER visits rates for males and females in Taiwan, 1998-2012.

Males

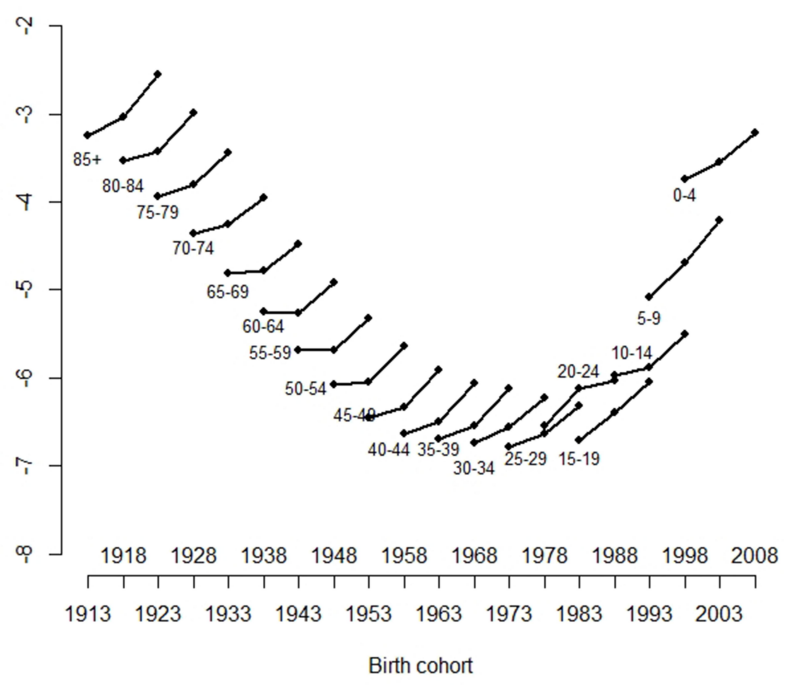

Females

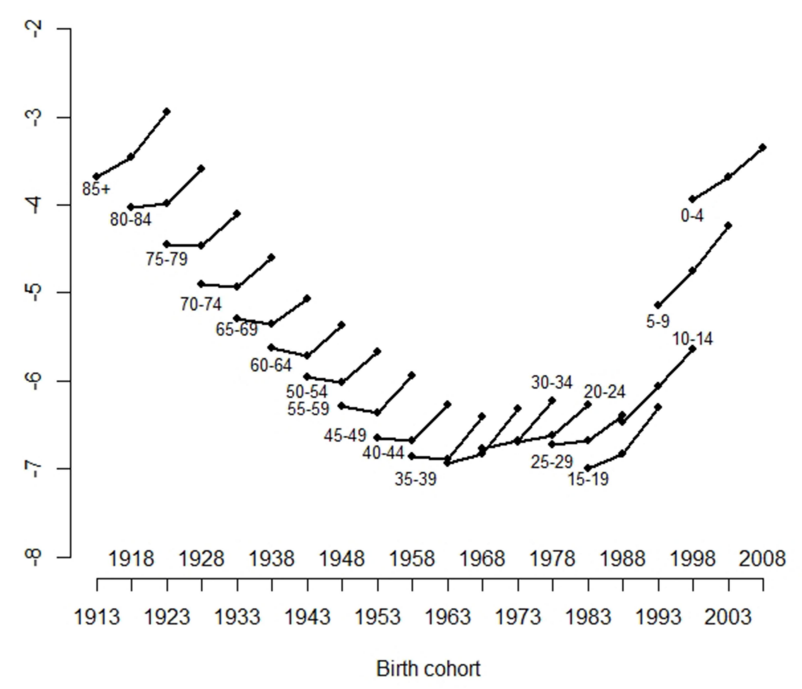

Figure 4 Age-specific ER visit rates of pneumonia-associated in log scale by birth cohort for males and females in Taiwan, 1998-2012.

physician capacity. To simplify the prediction procedure, we focus on the linear-extrapolated period effects for prediction of the next 5-year time period (ie, 2013-2017). Due to age effect is an integrated estimation from 15 years pneumoniarelated ER visit data, age effects still suit all age groups of the next time period, 2013-2017. Perdition of the next cohort effects (ie, 2013 birth cohort) conservatively keeps its value as the same as the 2008 birth cohort estimated value. During an extended time period such as 2013-2017, the average capacity for pneumonia-related visit to ER physician is about 82 under above prediction procedure. The increased number of pneumonia cases may be attributed to the increased imaging diagnosis in ER. Using the lung X-ray or $\mathrm{CT}$, if the ER physician finds an increase in infiltration with the patients, then these patients should have carefully diagnosis as a case of pneumonia. Moreover, Taiwan has been an aging society since 2018 . The elderly may have more risk of pneumonia-related visits in the ER. In the meantime, the emergent rescuer responsive hospitals must implement relevant acute care or healthcare because of requirements by the MOHW -namely, the Grading Responsible Hospitals for Acute Care (GRHAC) audit program, which was 
implemented from 2007 to 2009. The number of ER physicians had a dramatically increased trend since 2003 in response to the GRHAC audit program. Therefore, the average capacity for pneumonia-related visits to the ER physician had a decreased trend since 2003.

However, to our knowledge, no previous studies have separated pneumonia visit rates into age, period, and cohort effects or examined the influences on subsequent undiscussed to pneumonia in pediatric and older patients. The age, period, and cohort effects were slightly different between men and women. With regard to public health perspectives on gender, the incidence of some infectious diseases differs between men and women. For example, female adults have a higher incidence of urinary tract infection than male adults do. However, the samples used in these studies were too small, making it difficult to examine the differences by sex. Generally, our study showed that pneumonia visit rates did not differ by sex, and this result is somewhat consistent with that of previous studies. ${ }^{15,16}$

Although these interesting results should be paid much attention, there are some potential limitations that also need to be kept in mind. First, this research is a descriptive study and is considered an inferred phenomenon as the etiology is based on the changes in visit rates. The three effects in the APC model may be attributed to the differences in visit rates between groups caused by a major event. However, APC analysis based on longitudinal cohort information depends on certain development levels of countries. The approach to patients in the ER did not involve any symptoms of possible pneumonia in this study. Second, the APC models did not involve the adjustment of any confounding factors from aggregated format datasets, such as comorbidities, education levels, or lifestyles. In addition, the risk factors (comorbidities) for pneumonia were not available in aggregated format datasets. Fourth, the age-standardized rates for pneumonia increased during the study period (Figure 5), as reflected in the period effects. There have been general changes in medical technology and practices, specifically the improvements in the ability to detect bacteremia, ${ }^{19}$ which likely resulted in the increasing trends in pneumonia. The major advantage of the APC method is that it is not necessary to assume some systematic patterns of effects over the linear regression model. Moreover, it would be important to perform subgroup analyses according to the etiology of pneumonia and the severity of the patients when they consulted in the ER. However, the etiology and severity measurements for pneumonia were not available in aggregated format datasets.

The interpretation of our findings is presented in the next paragraph. This study showed that age is a risk factor for pneumonia, especially for children and adolescents and older patients. ${ }^{20}$ In that case, we might have expected similar patterns for septicemia (as an infectious disease), which was evident. $^{21}$ The case-fatality rate (CFR) was lowest in the youngest group (birth through 9 years). According
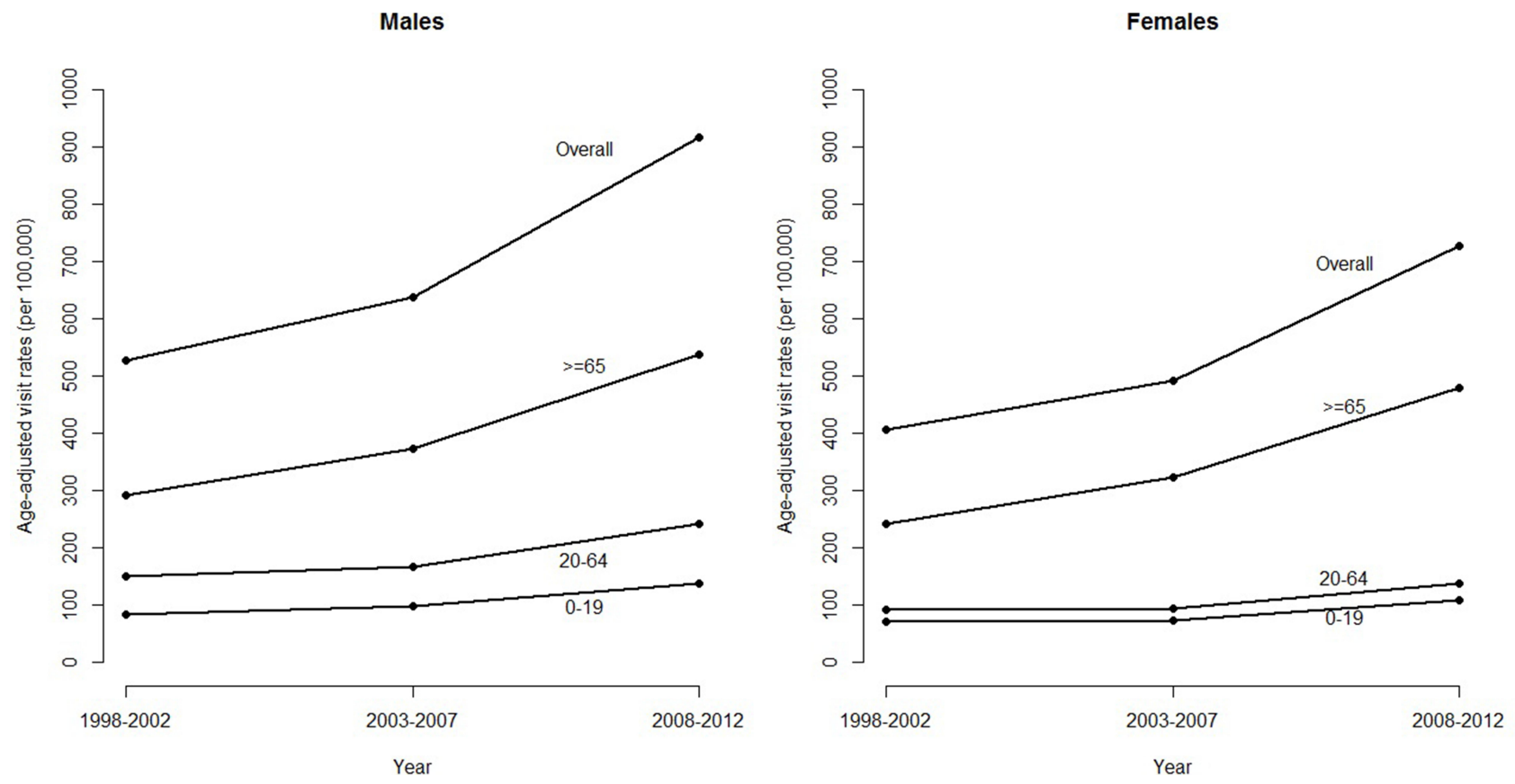

Figure 5 Observation of age-adjusted visit rates from pneumonia for stratified age groups (includes 0-19 years, 20-64 years, and 65+ years). 
to a report on the epidemiology of invasive pneumococcal disease among 2008-2013 from Taiwan Centers for Disease Control, the average CFR was $18.2 \%$ and the CFR of cases in the $\geq 75$ years age group was $35.5 \%$. Besides, CFR of bacteremic infections increased with each decade of life, which may attribute to a cohort effect. We also investigated cohort effects which may affect pneumonia visit rates in the ER for both genders. This age effect might be attributed to the adolescent and older patients' weak resistance to diseases. ${ }^{20}$ We suggest that ER physicians focus more attention to pediatric and older patients with suspected pneumonia. The gender difference in pneumonia visit rates was reported in previous studies. ${ }^{22}$ However, gender-specific psychosocial responses at sensitive developmental disease phases (ie, during childhood or puberty) could potentially focus development of the immune system for a specific gender effect. ${ }^{23}$ Our findings also coincide with evidence showing that better early life conditions may enhance immunity against bacterial infections, especially in females. Period effects should be treated as a serious issue on evolution of bacteremia detection. ${ }^{24} \mathrm{~A}$ more sensitive method for detection of bacteremia was developed for infectious diseases. The cohort effect oscillates among the birth cohort year. The recent cohort had higher pneumonia visit rates than the older cohort. A population-based retrospective cohort study shows that pneumococcal polysaccharide vaccine can only reduce the older adults' mortality. ${ }^{25}$ We may also attribute this to the fact that evolving mutations of pneumococcus seem to test the therapeutic effect of antibiotics. The outer layer of the pneumococcus has a transparent capsule, which will block the phagocytosis of human leukocytes so that it can have plenty of time to replicate in large quantities. Bacteria only need a short time to reproduce and generate a new generation. During the reproduction process, mutations are non-susceptible to antibiotics (ie, drug-resistant strains) new generation. After selection of antibiotics, non-resistant strains were gradually eliminated. But drug-resistant strains gradually grew and began to spread. This phenomenon may result in the $\mathrm{N}$ shape of the cohort effect, especially as the recent cohort had higher pneumonia visit rates than the older cohort (Figure 3). In this study, we successfully identify age, period, and cohort effects of pneumonia visit rates in ER used APC analysis. This study also added to understanding that the ER physicians' capacity to supply respiratory diseases care services over time is a key element in the process of planning for the future.

\section{Conclusion}

In conclusion, we found that the trend in pneumonia visit rates can be estimated by age, period, and cohort effects. Age is a risk factor for pneumonia-related ER visits, especially for children and adolescents and older patients. Period and cohort effects were also found to increase the pneumonia visit rates.

\section{Abbreviations}

APC, age-period-cohort; CFR, case-fatality rate; ER, emergency rooms; EU, Europe Union; HAdV, human adenovirus; HPIV, human parainfluenza virus; HRV, human rhinovirus; ICD, International Classification of Disease; MOHW, Ministry of Health and Welfare; RSV, respiratory syncytial virus.

\section{Data Sharing Statement}

The datasets used analyzed during the current study are available from the corresponding author on reasonable request by emailing istzeng@gmail.com.

\section{Ethics Approval and Consent to Participate}

The present study was approved by the Institutional Review Board of Chang Gung Memorial Hospital.

\section{Consent for Publication}

Not applicable in this study due to secondary data analysis.

\section{Author Contributions}

All authors made a significant contribution to the work reported, whether that is in the conception, study design, execution, acquisition of data, analysis and interpretation, or in all these areas; took part in drafting, revising or critically reviewing the article; gave final approval of the version to be published; have agreed on the journal to which the article has been submitted; and agree to be accountable for all aspects of the work.

\section{Funding}

No funding supports this study.

\section{Disclosure}

The authors report no competing interests with any organization with a direct or indirect financial interest in the subject matter discussed in the manuscript. 


\section{References}

1. Davidson MW, Haim DA, Radin JM. Using networks to combine "big data" and traditional surveillance to improve influenza predictions. Sci Rep. 2015;5(1):8154. doi:10.1038/srep08154

2. Ishiguro T, Kagiyama N, Uozumi R, et al. Clinical characteristics of influenza-associated pneumonia of adults: clinical features and factors contributing to severity and mortality. Yale J Biol Med. 2017;90 (2):165-181.

3. Marshall DC, Goodson RJ, Xu Y, et al. Trends in mortality from pneumonia in the Europe union: a temporal analysis of the European detailed mortality database between 2001 and 2014. Respir Res. 2018;19(1):81. doi:10.1186/s12931-018-0781-4

4. Fry AM, Shay DK, Holman RC, Curns AT, Anderson LJ. Trends in hospitalizations for pneumonia among persons aged 65 years or older in the United States, 1988-2002. JAMA. 2005;294(21):2712-2719. doi:10.1001/jama.294.21.2712

5. Lim TK, Siow WT. Pneumonia in the tropics. Respirology. 2018;23 (1):28-35. doi:10.1111/resp.13137

6. McAllister DA, Liu L, Shi T, et al. Global, regional, and national estimates of pneumonia morbidity and mortality in children younger than 5 years between 2000 and 2015: a systematic analysis. Lancet Glob Health. 2019;7(1):e47-e57. doi:10.1016/S2214-109X(18)30408-X

7. Berg AS, Inchley CS, Fjaerli HO, Leegaard TM, Nakstad B. Assessing severity in pediatric pneumonia: predictors of the need for major medical interventions. Pediatr Emerg Care. 2017. doi:10.1097/PEC.0000000000001179

8. Bhuiyan MU, Snelling TL, West R, et al. Role of viral and bacterial pathogens in causing pneumonia among Western Australian children: a case-control study protocol. BMJ Open. 2018;8(3):e020646. doi:10.1136/bmjopen-2017-020646

9. Müller-Redetzky H, Lienau J, Suttorp N, Witzenrath M. Therapeutic strategies in pneumonia: going beyond antibiotics. Eur Respir Rev. 2015;24(137):516-524. doi:10.1183/16000617.0034-2015

10. Ministry of Health and Welfare, R.O.C. National Health Insurance Health Statistics Annual Report.

11. Simões TC, Borges LF, Parreira de Assis AC, et al. Chagas disease mortality in Brazil: A Bayesian analysis of age-period-cohort effects and forecasts for two decades. PLoS Negl Trop Dis. 2018;12(9): e0006798. doi:10.1371/journal.pntd.0006798

12. Krieger N, Williams DR. Changing to the 2000 standard million: are declining racial/ethnic and socioeconomic inequalities in health real progress or statistical illusion? Am J Public Health. 2001;91 (8):1209-1213. doi:10.2105/AJPH.91.8.1209

13. $\mathrm{R}$ Development Core Team. $R:$ A Language and Environment for Statistical Computing. Vienna, Austria: R Foundation for Statistical Computing; 2008. ISBN 3-900051-07-0: http://www.R-project.org. Accessed July 31, 2020.

14. Cameron AC, Trivedi PK. Regression-based tests for overdispersion in the Poisson model. $J$ Econom. 1990;46(3):347-364. doi:10.1016/ 0304-4076(90)90014-K

Risk Management and Healthcare Policy

\section{Publish your work in this journal}

Risk Management and Healthcare Policy is an international, peerreviewed, open access journal focusing on all aspects of public health, policy, and preventative measures to promote good health and improve morbidity and mortality in the population. The journal welcomes submitted papers covering original research, basic science, clinical \& epidemiological studies, reviews and evaluations,
15. Neuman MI, Ting SA, Meydani A, Mansbach JM, Camargo CA Jr. National study of antibiotic use in emergency department visits for pneumonia, 1993 through 2008. Acad Emerg Med. 2012;19 (5):562-568. doi:10.1111/j.1553-2712.2012.01342.x

16. Neuman MI, Shah SS, Shapiro DJ, Hersh AL. Emergency department management of childhood pneumonia in the United States prior to publication of national guidelines. Acad Emerg Med. 2013;20 (3):240-246. doi:10.1111/acem.12088

17. Shah S, Bourgeois F, Mannix R, Nelson K, Bachur R, Neuman MI. Emergency department management of febrile respiratory illness in children. Pediatr Emerg Care. 2016;32(7):429-434. doi:10.1097/ PEC.0000000000000721

18. Buppajarntham A, Apisarnthanarak A, Khawcharoenporn T, Rutjanawech S, Singh N. National survey of Thai infectious disease physicians on treatment of carbapenem-resistant acinetobacter baumannii ventilator-associated pneumonia: the role of infection control awareness. Infect Control Hosp Epidemiol. 2016;37(1):61-69. doi:10.1017/ ice.2015.240

19. Livache T, Boisset S, Maurin M, Slimani S, Mathey R, Roupioz Y. Biochips for direct detection and identification of bacteria in blood culture-like conditions. Sci Rep. 2017;7(1):9457. doi:10.1038/s41598017-10072-z

20. Cascini S, Kirchmayer U, Belleudi V, et al. Inhaled corticosteroid use in chronic obstructive pulmonary disease and risk of pneumonia: a nested case-control population-based study in lazio (Italy)-The OUTPUL Study. COPD. 2017;14(3):311-317. doi:10.1080/15412555.2016.1254172

21. McGowan JE, Barnes MW, Finland M. Bacteremia at Boston City Hospital: occurrence and mortality during 12 selected years (1935-1972), with special reference to hospital-acquired cases. J Infect Dis. 1975;132(3):316-335. doi:10.1093/infdis/132.3.316

22. Auerbach O, Stout AP, Hammond EC, Garfinkel L. Changes in bronchial epithelium in relation to sex, age, residence, smoking and pneumonia. N Engl J Med. 1962;267(3):111-119. doi:10.1056/ NEJM196207192670301

23. Wong I, Cowling BJ, Leung GM, Schooling CM. Trends in mortality from septicaemia and pneumonia with economic development: an age-period-cohort analysis. PLoS One. 2012;7(6):e38988. doi:10. 1371/journal.pone. 0038988

24. Kim L, McGee L, Tomczyk S, Beall B. Biological and epidemiological features of antibiotic-resistant streptococcus pneumoniae in pre- and post-conjugate vaccine eras: A United States perspective. Clin Microbiol Rev. 2016;29(3):525-552. doi:10.1128/CMR.00058-15

25. Kolditz M, Schmitt J, Pletz MW, Tesch F. Impact of pneumococcal polysaccharide vaccine on incidence and mortality after pneumonia in adults aged $\geq 60$ years - a population-based retrospective cohort study. Clin Microbiol Infect. 2017;24:250. guidelines, expert opinion and commentary, case reports and extended reports. The manuscript management system is completely online and includes a very quick and fair peer-review system, which is all easy to use. Visit http://www.dovepress.com/testimonials.php to read real quotes from published authors. 